\title{
Application of Surface Topological Segmentation to Seismic Imaging
}

\author{
Timothée Faucon $^{1,2}$, Etienne Decencière ${ }^{1}$, and Cédric Magneron ${ }^{2}$ \\ ${ }^{1}$ Centre de Morphologie Mathémathique \\ Ecole des Mines de Paris, \\ 35 rue Saint Honoré, \\ 77305 Fontainebleau, France \\ \{timothee.faucon, etienne.decenciere\}@cmm.ensmp.fr \\ http://cmm.ensmp.fr \\ ${ }^{2}$ Earth Resource Management Services (ERM.S), \\ 16, rue du château, \\ 77300 Fontainebleau, France \\ \{timothee.faucon, cedric.magneron\}@erms.fr \\ http://www.erms.fr
}

\begin{abstract}
An original and efficient method to segment and label horizontal structures in $3 \mathrm{D}$ seismic images is presented. It is based on a morphological hierarchical segmentation. The initial extracted surfaces are post-processed using the topological segmentation method proposed by Malandain et al 1 . A last post-processing step allows to separate remaining multi-layered surfaces.
\end{abstract}

\section{Introduction}

Seismic imaging has become an essential technique in seismic exploration and exploitation. Thanks to it, industrialists have a better understanding of the subsoil structure and save time in seismic data processing. It also brings them a large panel of tools to improve their exploration and exploitation process. However, information is hidden in 3D seismic images and one of the prerequisites to benefit from the interesting information contained in the $3 \mathrm{D}$ data is to carry out several processings to adequately modify original data. One of these consists in extracting quasi horizontal structures also called horizons. They correspond to the sediment layers and are useful for geophysicists to locate oil and gas reservoirs. Horizon extraction might seem relatively easy. In fact, it is difficult due to the presence of geological faults, noise and acquisition artifacts. Moreover, it must be accurate since many applications depend on it. One of the other constraints in horizon extraction is that it is really time consuming and often requires a geoscientist expertise to be done.

The extraction method described here is an original way of picking and labelling horizons from seismic data. After briefly presenting seismic imaging in section 2, we proceed with the description of the picking method: in section 3 we 
describe briefly the horizon extraction part, which was presented in [2]. In section 4 we present the application of the surface segmentation method proposed by Malandain et al. 1] to our data. The result is not completly satisfactory in our framework due to surfaces composed of multiple layers. In section [5] a new method to solve this problem is introduced. Then before concluding on this work, we give some computation times to evaluate the efficiency of the processing chain.

\section{Seismic Imaging}

The first seismic data were acquired on tapes in a rudimentary way. Then, in the $60 \mathrm{~s}$, transistors and digital technologies appeared and allowed to sample the subsoil in 2D. The oil industry started to be interested in those methods of subsoil imaging to find and monitor oil fields. In the early 70 s the first 3D seismic survey was achieved on a field near Houston. 3D seismic acquisition has been since then considered as a reliable and cost-effective method of optimizing oil field exploration, development and management.

\subsection{Acquisition Mode}

Like echography in the medical field, seismic images are acquired through an indirect technique using acoustic waves. The acquisition process can be implemented offshore as well as onshore. In both cases it requires a sonar source (air-gun for marine acquisition and vibrators for ground one) which sends waves across the subsoil and receptors that register the signal reflected by the interfaces of sediment layers. This limit between two layers with different acoustic properties is called a seismic horizon. A large number of preprocessings are necessary to make the acquired data ready for analyses and interpretations by geoscientists. Those preprocessings provide the interpreters with many images. Each image is the representation of a seismic attribute which is defined by everything that can be computed from the original data or from other attributes [3]. Up to now, we have only worked on images of wave amplitudes. As shown in Fig. 1 amplitude images are a bright and dark layers stacking. These layers correspond to the sediment deposits. We call inline the direction of the acquisition system (given by the alignment of source and receptors) and cross-line the perpendicular direction to the inline one. These two directions correspond to the horizontal planes. The third dimension is called time (which can be converted into depth) and corresponds to the vertical direction perpendicular to the two others.

\subsection{Why Segmenting 3D Seismic Images?}

Segmenting 3D seismic images, i.e. extracting coherent seismic events (horizons), addresses several issues. A first family of these issues may be related to seismic processing. A set of coherent horizons is sometimes used as a guide within particular seismic processing steps. It allows to introduce geological information into classical seismic oriented processing. A second family is related to the time to 

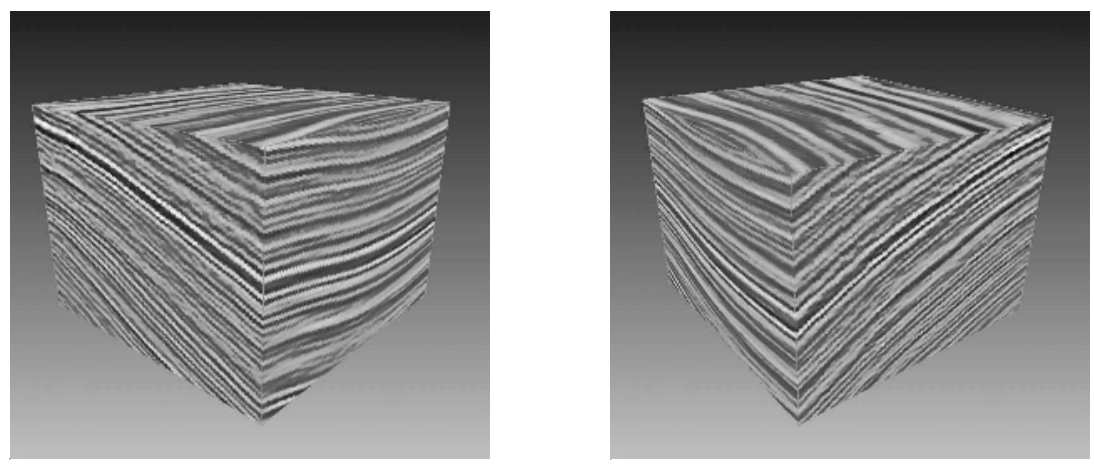

Fig. 1. Two views of the same 3D seismic cube used to illustrate this paper. Dimensions: $201 \times 201 \times 150$.

depth conversion process. Seismic images in time are converted in depth. Major identified horizons are useful to achieve this conversion. They result from a structural interpretation. A third family can be identified when dealing with reservoir modelling. Horizons at a small vertical scale result from a stratigraphic interpretation. Other operational applications may be found as segmenting 3D seismic images is a really new approach. Some techniques already exist to segment seismic images. Among those, we can find the ones which take into account the image structure 4] or use wavelets [5] to realize the segmentation. Some other techniques are based on the division of space into a set of meshes and on a pairing of similar meshes [6]. We also find seismic images segmentation using fuzzy techniques [7]. A little number of papers deal with seismic data processed by mathematical morphology techniques ( $[8,9]$ ). Thus segmenting 3D seismic images with mathematical morphology appears to be an innovative method.

\section{Seismic Horizon Extraction Using Morphological Segmentation}

Segmenting an image can be achieved in several ways. The watershed invented in 1979 by S. Beucher and Ch. Lantuéjoul [10] is a morphological solution and is based on an imaginary flooding of a topography usually given by the gradient of the original image. The flooding starts from sources determined by the user or another algorithm and produces a fine partition of the gradient image.

Because of the seismic image structure and of the horizon picking extraction application, we do not use the gradient but the original amplitude image as an entry to the watershed algorithm. The horizons we are looking for are the image brighter surfaces. With the watershed, we directly obtain the principal horizons that correspond to the maximal amplitudes. 


\subsection{Hierarchical Segmentation}

The fine segmentation of a seismic image gives however too many regions to be easily interpreted. To restrict the number of areas, we could have made a selection of the flooding sources but nothing can ensure that the brighter surfaces are situated between two selected minima. Another solution to reduce the final number of horizons, while preserving the more representative geological structures, is to select the best ones according to a certain criterion among the whole set of horizons. To reach this goal, we used the hierarchical segmentation introduced by F. Meyer in 1994 [11. This algorithm creates a graph associated to the image. Each graph node represents one of the image regions i.e. the influence zone of a minimum with respect to the topography. During the flooding process, each time two basins merge, the corresponding nodes are linked by an edge valuated with the smallest value of a criterion computed from the basins characteristics. This criterion can be the depth, the area or the volume of the basin. We finally obtain a minimum spanning tree. By cutting the $k-1$ bigger edges, we obtain from this tree $k$ regions, which are typically separated by the brightest horizons.

\subsection{Segmentation with Cylinders}

Some horizons that seem to be among the brightest ones do no appear in the results even if we ask for a large amount of horizons. This is due to some voxels of these horizons which have a lower gray level than the rest of the structure. These voxels lead to a "leak" during the flooding step and as the two bordering basins merge earlier than they should, the final surface appears lower in the hierarchy. To restrict this problem to the leak location and pick the rest of the horizon we used a method described in 2, [12. This method consists in dividing the original image into many subimages called cylinders and in computing a hierarchical segmentation inside each of them. The leak problem is thus restricted to a small number of cylinders and the major part of each brightest horizon appears in the resulting image. To preserve the picked structures continuity, we make the cylinders overlap.

Each time a pixel within a given cylinder is considered as belonging to a horizon (i.e. belongs to the watershed line), an accumulator image is incremented at the corresponding position. Once all cylinders have been segmented, the accumulator contains a grey level image. After thresholding, the resulting binary image corresponds to the extracted horizons. Figure 2 shows the result of the application of the horizon extraction method to our test cube, after labelling (26-connexity). It contains only three connected components.

In practice, most structures appearing in the accumulator are thin, but, for several reasons, this is not always the case. Therefore, a simple threshold is not enough to binarise the accumulator if we want to obtain thin surfaces.

\subsection{Thinning}

To implement our labelling algorithm and avoid the errors due to the thickness of surfaces, we need thin surfaces. That means that surfaces are expected to 


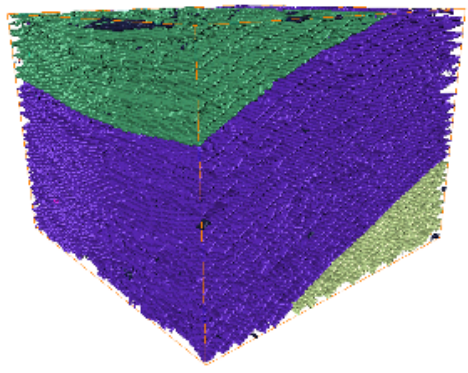

$3 \mathrm{D}$ view

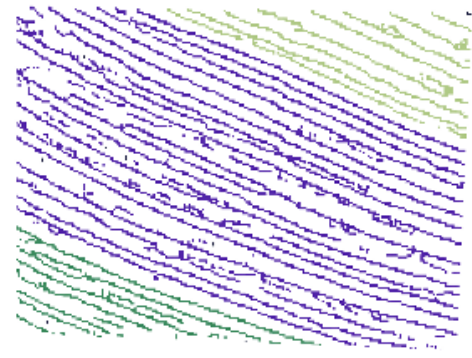

$2 \mathrm{D}$ section

Fig. 2. Labelled horizons extracted from the test cube, without post-processing

have a thickness of one voxel. We have used an a priori knowledge about the application for which we are trying to segment the image and we work with a binarisation of the accumulation image obtained from the morphological segmentation.

As we are trying to extract the brightest voxels, which characterise the structures we are looking for (Fig. 3a), we identify the connected voxels in a vertical neighborhood $(1 \times 1 \times 3)$ see Fig. 3 b. Then we eliminate those which have the lowest amplitude (Fig. 3r). In the particular case of slopes higher than forty five degrees, this technique can introduce horizontal holes into the surface but since we are working in 3D the surface connectivity is preserved. Given the nature of the images, such high slopes are very uncommon, however, further developments of this thinning process are envisaged and will lead to the correction of these drawbacks.

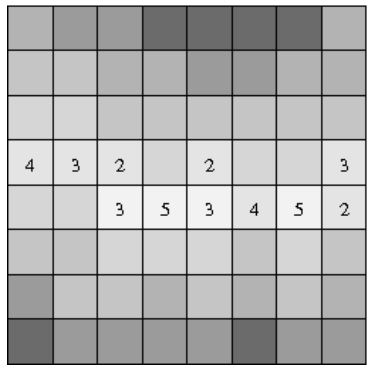

(a) Grey level image and accumulator values

\begin{tabular}{|l|c|c|c|c|c|c|c|}
\hline 179 & 155 & 155 & 107 & 107 & 107 & 107 & 179 \\
\hline 197 & 197 & 179 & 179 & 155 & 155 & 179 & 179 \\
\hline 214 & 214 & 197 & 197 & 197 & 197 & 197 & 197 \\
\hline 228 & 228 & 228 & 214 & 228 & 214 & 214 & 228 \\
\hline 214 & 214 & 242 & 242 & 242 & 228 & 242 & 220 \\
\hline 197 & 197 & 214 & 214 & 214 & 197 & 214 & 197 \\
\hline 155 & 197 & 197 & 179 & 197 & 179 & 197 & 179 \\
\hline 107 & 155 & 155 & 155 & 155 & 107 & 155 & 155 \\
\hline
\end{tabular}

(b) Grey level values

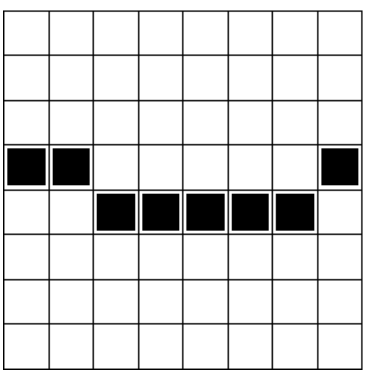

(c) Thinning result

Fig. 3. Thinning process 


\section{Topological Segmentation}

Superimposed horizons which are connected (i.e. surfaces in which some voxels have the same geographical coordinates) are a real problem for the interpretation and the other applications depending on geological structure picking. To address this problem, we took as a starting point the approach of Malandain et al. 1. This method creates a voxel classification using the topology of object and background voxel neighborhood.

\subsection{Voxel Classification}

Malandain et al. have implemented a voxel classification in order to segment surfaces in 3D images. Thus they have established two relevant numbers $C^{*}$ and $\bar{C}$ that respectively describe the object connectivity and the background connectivity in a given neighborhood. Considering an object $X$, its background $\bar{X}$ and a neighborhood $V$ of an object point $x$, the two numbers are:

$-C^{*}$ : number of 26-connected components of $X \cap V^{*} 26$-adjacent to $x$, and

- $\bar{C}$ : number of 6-connected components of $\bar{X} \cap V$-adjacent to $x$,

where $V^{*}$ is $V \backslash\{x\}$.

Connectivity and Neighborhoods. According to 13, object and background adjacency have to be different. Usually, adjacency used for the object is the 26- or 18- adjacency and the one used for the background is the 6-adjacency. Like Malandain et al., we have followed this convention.

According to the definition of $C^{*}$, the connected components of the object have to be 26 -adjacent to the central point $x$. Thus, using a 26 -connected neighborhood to compute $C^{*}$ allows to avoid the calculation of the 26 -adjacency. We differ from Malandain et al. in the neighborhood choice for the computation of $\bar{C}$. Indeed, we have chosen to compute it rather in a 26 -neighborhood than in a 18-neighborhood. This choice is motivated by an algorithmic reason. Computing $C^{*}$ and $\bar{C}$ in the same neighborhood simplifies and accelerates the computation.

The resulting values of $C^{*}$ and $\bar{C}$ according to their neighborhood are summarized in Table 1 .

Table 1. Class of point according to the value of $C^{*}$ and $\bar{C}$

\begin{tabular}{llll}
\hline Type A & interior point & $\bar{C}=0$ & \\
Type B & isolated point & & $C^{*}=0$ \\
Type C & border point & $\bar{C}=1$, & $C^{*}=1$ \\
Type D & curve point & $\bar{C}=1$, & $C^{*}=2$ \\
Type E & curve junction & $\bar{C}=1$, & $C^{*}>2$ \\
Type F & surface point & $\bar{C}=2$, & $C^{*}=1$ \\
Type G & surface-curve junction & $\bar{C}=2$, & $C^{*} \geq 2$ \\
Type H & surface junction & $\bar{C}>2$, & $C^{*}=1$ \\
Type I & surface-curve junction & $\bar{C}>2$, & $C^{*} \geq 2$ \\
\hline
\end{tabular}




\subsection{Misclassification}

Problem of Misclassification. After this topological classification, we select the voxels we are interested in. These voxels are $\mathrm{C}$ Type (border points) and F Type (surface points) ones. As we eliminate the other points, specially the junction points, we should have well disconnected and easy to label surfaces with a simple labelling algorithm. In fact, that does not occur like that, as shown in Fig. 4. Some horizons are correctly separated from the others but some surface junctions are not correctly detected. This phenomenon comes from some troubles in the detection of junction points. Some junctions, because of their thickness, are wrongly labelled. The two examples shown in Fig. 5 present two cases where the junction points are labelled as surface points (the two grids represent a slice ot the $3 \mathrm{D}$ object). In case $a, \bar{C}=0$ for the center point. Thus, this point is considered as an interior point. In case $b, \bar{C}=0$ and $C^{*}=1$ thus the four center points are misclassified as surface points.

Processing of Misclassified Points. To tackle this problem, we refer again to [1] where a characterisation of simple surfaces is explained. Considering a surface point $x, B_{x}$ and $C_{x}$ are the two connected components of $\bar{X} \cap N_{26}^{*} 6$ adjacent to $x$ included in $X$. Two surface points $x$ and $y$ are said to be in relation if there is a 26-path $\left(x_{0}, x_{1}, \ldots, x_{i}, \ldots, x_{n}\right)$ included in $X$ with $x_{0}=x$ and $x_{n}=y$ such that for $i \in[0, \ldots n-1]$ :

$-B_{x_{i}} \cap B_{x_{i+1}} \neq 0$ and $C_{x_{i}} \cap C_{x_{i+1}} \neq 0$, or

$-B_{x_{i}} \cap C_{x_{i+1}} \neq 0$ and $C_{x_{i}} \cap B_{x_{i+1}} \neq 0$.

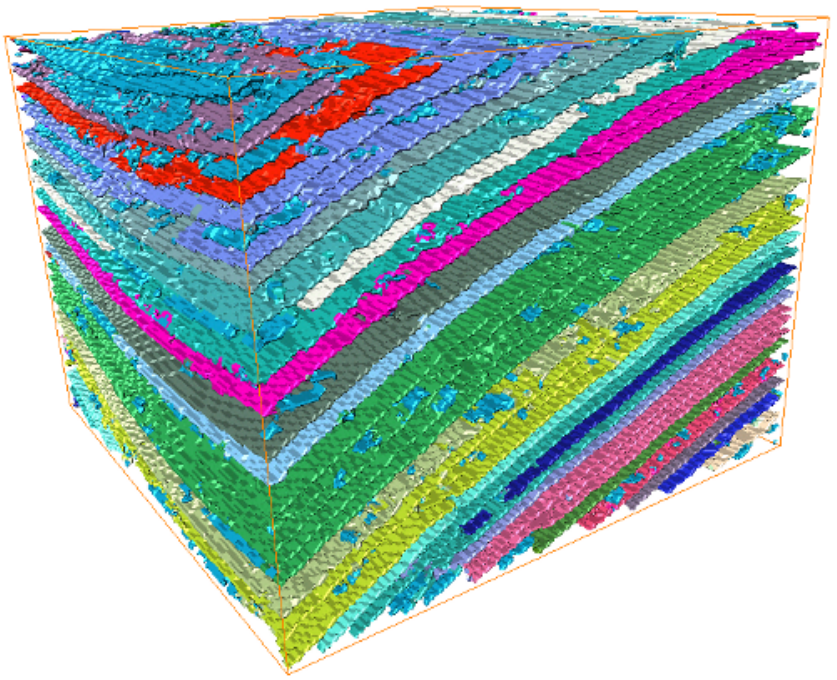

Image corrected from misclassified points

Fig. 4. Topological segmentation 

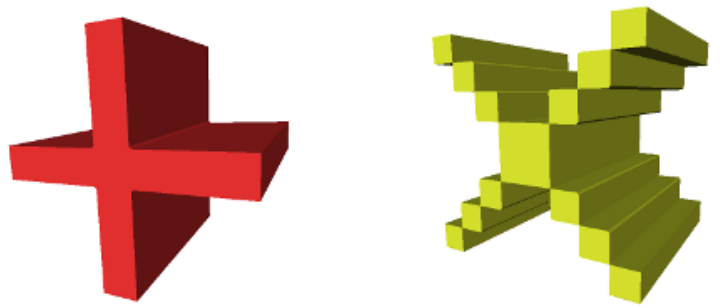

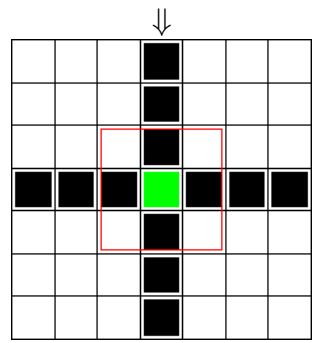

(a) $\bar{C}=0$ : the point is classified an interior point (Type A)

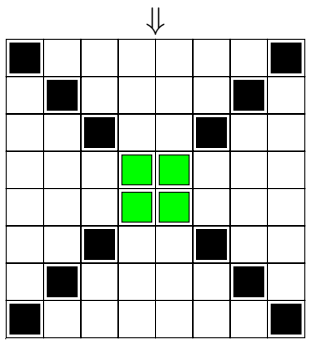

(b) $\bar{C}=2$ and $C^{*}=1$ : the point is classified as a surface point (Type F)

Fig. 5. Misclassification of junction points. Each image represents a slice of two different binary images constituted of a succession of these images.

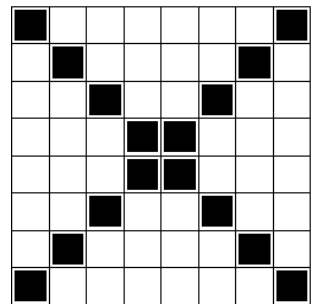

(a) Configuration example. All points are considered as surface points. The junction is not detected

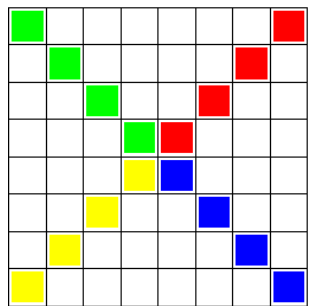

(b) Equivalence classes

Fig. 6. Solution for misclassification of junction points

This relation is an equivalence relation (it is reflexive, symmetric and transitive) and its equivalence classes compose therefore a partition of the surface points of $X$. Each region of the partition is a simple surface. In short, two surface 


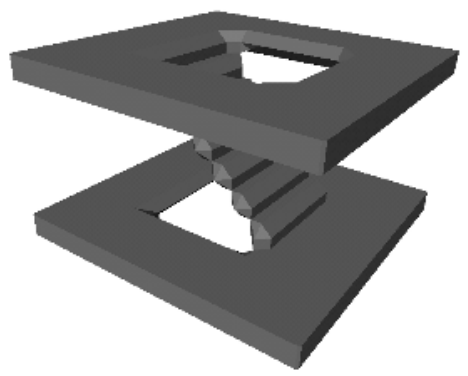

(a) Multi-layered surface

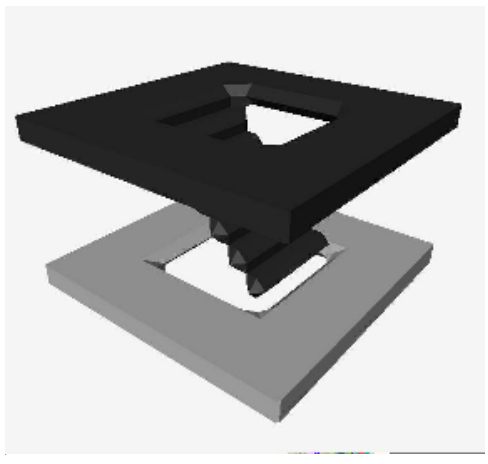

(b) Layers separation

Fig. 7. Layers separation example

points belong to the same surface if there is a succession of connected surface points linking these two points and having their two neighborhood connected components sharing at least one point pair by pair. This is illustrated in Fig. [6.

This post-processing is applied only to surface points which are the only ones to be misclassified. We can thus restrict the number of processed points. However, during the processing, border points are considered and labelled too in order to be integrated to the surface but they are not processed as the surface points. The results are shown in Fig. $4 \mathrm{~b}$ ). At the end of the processing, all points are correctly classified but some connected horizons still remain. The reasons are no more local problems but global and structural reasons: configurations such as the one shown in Fig. 7 are possible causes. We have first implemented this classification technique. Some other exist ( [14) but since the results of the presented method are satisfactory, they have not been implemented.

\section{Multiple Layers Separation}

\subsection{Why Separating Linked Surfaces?}

From a geological point of view, differenciating sediments layers is interesting especially if they are correctly separated even if a natural phenomenon (erosion, faults, etc...) occured and disturbed the geological deposition structure. At this step of our processing chain, some surfaces are still connected whereas they should not. We say that a surface is made of several layers if two points of this surface have the same geographical coordinates (inline and crossline). The way of separating the different structure layers is described below.

In the example of Fig. $7 \mathrm{~h}$ all the voxels are considered as border or surface voxels and they belong to the same connected component. They will thus receive the same label. This kind of configuration can be due to a fuzzy part of the image which implies a bad segmentation area. The resulting segmentation leads to this 
link between two surfaces which are not connected anywhere else. To solve this problem, we realize a last processing.

\subsection{Structure Separation}

To separate the linked surfaces, we have to give to each component of the multiple-layer surface a different label. Our new labelling process consists in maintaining a map of the current processed horizon (made of multiple layers) and to make a selection among the voxels candidates for stacking into the queue. Those for which there is already a voxel with the same geographical coordinates in the queue are not stacked in the same queue because they belong to another surface. Once the current queue is empty, we process the other ones. A point can be queued several times but it will never be labelled twice because a tag checking is done as it is pushed back from queue.

This leads to the results shown in Fig. 7b where the two different surfaces are separated.
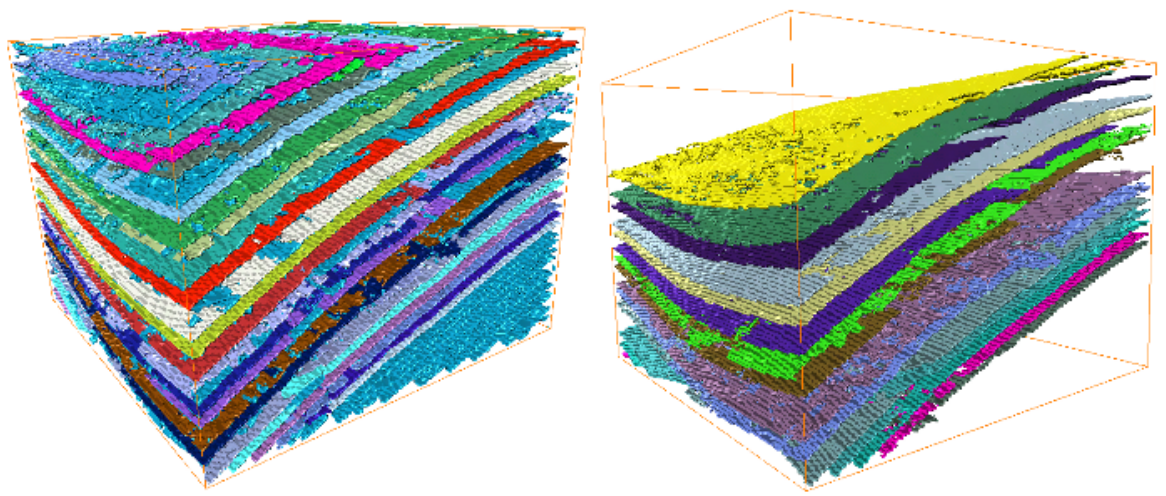

(a) Horizons correctly labelled (The multiple layer structure on the bottom right of the image is due to the visualisation software colormap

(b) With area filtering (elimination of small surfaces)

capabilities (only 256 levels).)

Fig. 8. Final labellisation

According to specialists, final result is very interesting. The surfaces extracted from the original data follow the maxima values of the geological structures and the surfaces are correctly separated. These results are shown in Fig. 8

\section{Computation Times}

The algorithms have been tested on a computer equipped with an Intel Pentium IV $3 \mathrm{GHz}$ and $1 \mathrm{~Gb}$ of RAM. The test image is an 8 bits grey level image with 
8 million points $(501 \times 400 \times 400)$. With these elements, the computation times are the following:

- The segmentation with cylinders takes about 3.5 hours with $501 \times 10 \times 10$ cylinders (i.e. 44100 cylinders) and with a step of one pixel in both directions. We have asked for forty regions in each cylinder (this parameter does not change the final computation time). Since each cylinder can be processed independently, this algorithm is strongly parallelizable.

- Topological surface labelling takes about forty minutes. This duration is not really representative because it depends directly on the number of required regions at the segmentation step i.e. the number of points contained in the object.

- Multi layer structure elimination. This section depends completely on the number of object points and the complexity of the structures. In our example its computation takes about 20 minutes.

Most of these times could be considerably improved through code optimization. However, the industrial partner of this study did not consider it a priority.

\section{Conclusion}

Segmenting and labelling 3D seismic images is a challenge. Both the 3D nature and the very large data volume represent major constraints for segmentation techniques to overcome. Our approach based on watershed segmentation applied to local volumes allows to derive fast segmentation results corresponding to a set of coherent seismic surfaces called horizons. Our labelling algorithms enable to individualize efficiently theses horizons in reasonable delays. As a conclusion, our segmentation/labelling solution is operational when applied to large seismic data volumes from a computation time point of view. Additional work, consisting in analysing the quality of the provided results, is planned in order to definitely validate the approach. Afterwards, several operational applications like geology guided seismic processing, structural interpretation, stratigraphic interpretation, etc. should benefit from it.

\section{References}

1. Malandain, G., Bertrand, G., Ayache, N.: Topological segmentation of discrete surfaces. International Journal of Computer Vision 10(2) (1993) 183-197

2. Faucon, T., Decencière, E., Magneron, C.: Morphological segmentation applied to 3D seismic data. In Ronse, C., Najman, L., Decencière, E., eds.: Mathematical Morphology: 40 Years On. Computational Imaging and Vision (2005) 475-484

3. Taner, M.: Attributes revisited. Rock Solid Image Houston Texas (1992)

4. Bakker, P.: Image structure analysis for seismic interpretation. PhD thesis, Technische Universiteit Delft (2002)

5. Bouchereau, I.B.: Analyse d'images par transformée en ondelettes; application aux images sismiques. PhD thesis, Université Joseph Fourier Grenoble (1997) 
6. Hale, D., Emmanuel, J.: Seismic interpretation using global image segmentation. 73th Annual International Meeting, Society of Exploration Geophysicists (2003)

7. Valet, L., Mauris, G., Bolon, P., Keskes, N.: Seismic image segmentation by fuzzy fusion of attributes. IEEE Transactions on Instrumentation and Measurement $\mathbf{5 0}$ (2001) 1014-1018

8. Moueddene, K.: Analyse d'images en sismique : pretraitement et extraction d'informations par la morphologie mathématique. PhD thesis, Université Paul Sabatier, Toulouse, France (1987)

9. N'Guyen, M.: Analyse multi-dimensionnelle et analyse par les ondelettes des signaux sismiques. PhD thesis, Institut National Polytechnique de Grenoble (2000)

10. Beucher, S., Lantuéjoul, C.: Use of watersheds in contour detection. In: International workshop on image processing, real-time edge and motion detection. (1979)

11. Meyer, F.: Minimal spanning forests for morphological segmentation. In Serra, J., Soille, P., eds.: Mathematical Morphology and its applications to signal processing (Proceedings ISMM'94), Fontainebleau, France, Kluwer Academic Publishers (1994)

12. S.Beucher, Decencière, E., Sandjivy, L., Magneron, C., Faucon, T.: (Demande de brevet français no 0503793 pour un procédé de détermination hiérarchique d'événements cohérents dans une image sismique)

13. Kong, T., Rosenfeld, A.: Digital topology: Introduction and survey. Computer Vision, Graphics, And Image Processing 48(1) (1989) 357-393

14. Svensson, S., Nyström, I., di Baja, G.S.: Curve skeletonization of surface-like objects in 3d images guided by voxel classification. Pattern Recognition Letter 23 (2002) 1419-1426 